\title{
EULER CHARACTERISTICS IN RELATIVE K-GROUPS
}

\author{
M. FLACH
}

\section{Introduction}

Suppose that $M$ is a finite module under the Galois group of a local or global field. Ever since Tate's papers $[\mathbf{1 7}, \mathbf{1 8}]$, we have had a simple and explicit formula for the Euler-Poincare characteristic of the cohomology of $M$. In this note we are interested in a refinement of this formula when $M$ also carries an action of some algebra $\mathscr{A}$, commuting with the Galois action (see Proposition 5.2 and Theorem 5.1 below). This refinement naturally takes the shape of an identity in a relative K-group attached to $\mathscr{A}$ (see Section 2). We shall deduce such an identity whenever we have a formula for the ordinary Euler characteristic, the key step in the proof being the representability of certain functors by perfect complexes (see Section 3). This representability may be of independent interest in other contexts.

Our formula for the equivariant Euler characteristic over $\mathscr{A}$ implies the isogeny invariance' of the equivariant conjectures on special values of the L-function put forward in [3], and this was our motivation to write this note. Incidentally, isogeny invariance (of the conjectures of Birch and Swinnerton-Dyer) was also a motivation for Tate's original paper [18]. I am very grateful to J-P. Serre for illuminating discussions on the subject of this note, in particular for suggesting that I consider representability. I should also like to thank D. Burns for insisting on a most general version of the results in this paper.

\section{The relative $K_{0}$}

We fix a prime number $p$ and a $\mathbb{Z}_{p}$-algebra $\mathscr{A}$ (associative with unit) which is either finite or finitely generated and free as a $\mathbb{Z}_{p}$-module. Let $K_{0}(\mathscr{A})$ (respectively $K_{0}\left(\mathscr{A}, \mathbb{Q}_{p}\right)$ ) be the Grothendieck group of the exact category $H(\mathscr{A})$ (respectively $H F(\mathscr{A})$ ) of finitely generated (respectively finite) $\mathscr{A}$-modules of finite projective dimension. For an object $M$ of $H F(\mathscr{A})$, we denote its class by $[M]_{\mathscr{A}} \in K_{0}\left(\mathscr{A}, \mathbb{Q}_{p}\right)$, and for a bounded complex $M^{\bullet}$ of objects of $H F(\mathscr{A})$, we put $[M]_{\mathscr{A}}=\sum(-1)^{i}\left[M^{i}\right]_{\mathscr{A}}$. We have a long exact localization sequence

$$
K_{1}(\mathscr{A}) \longrightarrow K_{1}\left(\mathscr{A}_{\mathbb{Q}}\right) \longrightarrow K_{0}\left(\mathscr{A}, \mathbb{Q}_{p}\right) \longrightarrow K_{0}(\mathscr{A}) \longrightarrow K_{0}\left(\mathscr{A}_{\mathbb{Q}}\right),
$$

where $\mathscr{A}_{\mathbb{Q}}:=\mathscr{A} \otimes_{\mathbb{Z}_{p}} \mathbb{Q}_{p}$. This is [1, Theorem IX.6.3] if $\mathscr{A}$ is a free $\mathbb{Z}_{p}$-module, whilst if $\mathscr{A}$ is Artinian, then obviously $K_{0}\left(\mathscr{A}, \mathbb{Q}_{p}\right)=K_{0}(\mathscr{A})$ and $K_{i}\left(\mathscr{A}_{\mathbb{Q}}\right)=0$.

Received 15 March 1998; revised 23 April 1999.

2000 Mathematics Subject Classification 19A99, 18G99, 11R34.

The author was supported by NSF-grant DMA-9624824 and a Sloan fellowship.

Bull. London Math. Soc. 32 (2000) 272-284 
EXAMPLES. (1) If $\mathscr{A}=\mathbb{Z}_{p}$, then $H F(\mathscr{A})$ consists of all finite $\mathbb{Z}_{p}$-modules. We write $[M]$ for $[M]_{\mathbb{Z}_{p}}$. There is an isomorphism $K_{0}\left(\mathscr{A}, \mathbb{Q}_{p}\right) \cong \mathbb{Z}$ which can be chosen so that $\# M=p^{[M]}$ for any finite $\mathbb{Z}_{p}$-module $M$. More generally, if $\mathscr{A}$ is Dedekind, then $K_{0}\left(\mathscr{A}, \mathbb{Q}_{p}\right)$ is the group of divisors on $\operatorname{Spec}(\mathscr{A})$ or, equivalently, of fractional $\mathscr{A}$-ideals, and $[M]_{\mathscr{A}}$ is the Fitting ideal of (any finite $\mathscr{A}$-module) $M$.

(2) For $\mathscr{A}_{\mathbb{Q}}$ a semisimple $\mathbb{Q}_{p}$-algebra, it is shown in [3, Section 2.2] how to construct elements in $K_{0}\left(\mathscr{A}, \mathbb{Q}_{p}\right)$ from a perfect complex $P \cdot$ of $\mathscr{A}$-modules together with a trivialization, that is, an isomorphism $\bigoplus_{i \text { even }} H^{i}\left(P_{\mathbb{Q}}\right) \stackrel{\sim}{\longrightarrow} \bigoplus_{i \text { odd }} H^{i}\left(P_{\mathbb{Q}}\right)$ where $P_{\dot{\mathbb{Q}}}=P^{\bullet} \otimes_{\mathbb{Z}_{p}} \mathbb{Q}_{p}$. This construction is crucial in formulating conjectures on special values of equivariant L-functions of motives whose $p$-adic realization carries an action of $\mathscr{A}_{\mathbb{Q}}$. The isogeny invariance of these conjectures will follow from Theorem 5.1 below.

(3) Let $N$ be a finite abelian $p$-group, say of order $p$, and let $\mathscr{A}=\mathbb{Z}_{p} \oplus N$ where $n m=0$ for all $n, m \in N$. This algebra does not satisfy our running assumptions, and it is indeed not hard to verify that $K_{0}\left(\mathscr{A}, \mathbb{Q}_{p}\right)=0$ (with our definition above) whereas any group $K_{0}\left(\mathscr{A}, \mathbb{Q}_{p}\right)$ fitting into a long exact sequence (1) has to be nonzero.

Remark 2.1. Consider triples $(X, g, Y)$, where $X$ and $Y$ are finitely generated projective $\mathscr{A}$-modules, and $g: X \otimes_{\mathbb{Z}_{p}} \mathbb{Q}_{p} \longrightarrow Y \otimes_{\mathbb{Z}_{p}} \mathbb{Q}_{p}$ is an $\mathscr{A}_{\mathbb{Q}}$-isomorphism. There is a rather different description of $K_{0}\left(\mathscr{A}, \mathbb{Q}_{p}\right)$ as the group generated by such triples together with certain relations [1]. If one defines $K_{0}\left(\mathscr{A}, \mathbb{Q}_{p}\right)$ in this way, then the sequence (1) holds true in Example (3), and in fact for an arbitrary ring homomorphism in place of $\mathscr{A} \longrightarrow \mathscr{A}_{\mathbb{Q}}$. This description of $K_{0}\left(\mathscr{A}, \mathbb{Q}_{p}\right)$ is also crucial for the constructions of Example (2), but it is of little use for the purposes of this paper.

Remark 2.2. Let $D^{\text {perf }}(\mathscr{A})$ (respectively $D^{f \operatorname{perf}}(\mathscr{A})$ ) be the full triangulated subcategory of the derived category of (left) $\mathscr{A}$-modules consisting of complexes quasi-isomorphic to a bounded complex of finitely generated projective $\mathscr{A}$-modules (and with finite cohomology). Any bounded complex of objects of $H(\mathscr{A})$ (respectively $H F(\mathscr{A})$ ) is an object of $D^{\text {perf }}(\mathscr{A})$ (respectively $D^{f \text { perf }}(\mathscr{A})$ ). There is a notion of $K_{0}$ of a triangulated category [10, Exposé VIII], and one can show that $K_{0}(\mathscr{A}) \cong K_{0}\left(D^{\text {perf }}(\mathscr{A})\right)$ [10, Section 7]. Using, for example, the results of [19], one can also show that $K_{0}\left(\mathscr{A}, \mathbb{Q}_{p}\right) \cong K_{0}\left(D^{f \operatorname{perf}}(\mathscr{A})\right)$. In some sense this is the most natural point of view on $K_{0}\left(\mathscr{A}, \mathbb{Q}_{p}\right)$ for the purposes of this paper, because we shall be interested in the classes of cohomology complexes which happen to be quasiisomorphic to bounded complexes of objects of $H F(\mathscr{A})$, but which are naturally determined only up to quasi-isomorphism in the derived category of all $\mathscr{A}$-modules.

\section{Representability}

Let $\mathscr{B}$ be a profinite $\mathbb{Z}_{p}$-algebra, and let $\mathscr{C}=\mathscr{C}(\mathscr{B})($ respectively $\mathscr{D}=\mathscr{D}(\mathscr{B}))$ be the category of profinite (respectively discrete) continuous $\mathscr{B}$-modules with continuous homomorphisms. For $M, N$ objects of either $\mathscr{C}$ or $\mathscr{D}$, we denote by $\operatorname{Hom}_{\mathscr{B}}^{c}(M, N) \subseteq$ $\operatorname{Hom}_{\mathscr{B}}(M, N)$ the set of continuous homomorphisms. $\mathscr{C}$ is an abelian category with enough projectives [2, Lemma 1.6], and contains all finitely generated continuous $\mathscr{B}$-modules. $\mathscr{C} \cap \mathscr{D}$ is the category of finite continuous $\mathscr{B}$-modules. There might exist finite, hence also finitely generated $\mathscr{B}$-modules on which the action of $\mathscr{B}$ is not 
continuous, but we shall never consider these. From now on we assume that all $\mathscr{B}$-modules are continuous, without further mention.

A well-known representability theorem of Grothendieck asserts that any left exact functor $F: \mathscr{C} \cap \mathscr{D} \longrightarrow \mathrm{Ab}$ is isomorphic to the functor $X \longmapsto \operatorname{Hom}_{\mathscr{B}}^{c}(M, X)$ for some object $M$ of $\mathscr{C}$ [7, Chapter V, Section 2, Theorem 3.1], [9]. $M$ is projective in $\mathscr{C}$ (respectively finitely generated) if and only if $F$ is exact [2, Proposition 3.1] (respectively satisfies a certain growth condition; see Lemma 3.3 below). Our aim in this section is to establish the following representability result for complexes.

Proposition 3.1. Assume that $M \longmapsto C \cdot(M)$ is a functor from $\mathscr{C} \cap \mathscr{D}$ to (cochain) complexes of $\mathbb{Z}_{p}$-modules such that:

(i) there exist $n, m \in \mathbb{Z}$ such that for all $M \in \mathrm{Ob}(\mathscr{C} \cap \mathscr{D}), H^{i}\left(C^{\bullet}(M)\right)=0$ for $i \notin[n, m]$;

(ii) there exists $a_{i} \geqslant 0$ such that for all $M \in \mathrm{Ob}(\mathscr{C} \cap \mathscr{D}), \# H^{i}(C \cdot(M)) \leqslant(\# M)^{a_{i}}$;

(iii) each $C^{i}$ is an exact functor in $M$.

Then there exists a bounded (chain) complex $P$. of finitely generated, projective $\mathscr{B}$ modules and a natural isomorphism in the derived category of $\mathbb{Z}_{p}$-modules

$$
\operatorname{Hom}_{\mathscr{B}}\left(P_{\bullet}, M\right) \stackrel{\sim}{\longrightarrow} C^{\bullet}(M) .
$$

Note that $\operatorname{Hom}_{\mathscr{B}}^{c}\left(P_{i}, M\right)=\operatorname{Hom}_{\mathscr{B}}\left(P_{i}, M\right)$, since $P_{i}$ is finitely generated. Conversely, if $P$. is such a complex, then the functor $\operatorname{Hom}_{\mathscr{B}}\left(P_{\bullet},-\right)$ satisfies (i), (ii) and (iii).

REMARK 3.1. If there are only finitely many simple $\mathscr{B}$-modules up to isomorphism, then by an easy dévissage argument, conditions (i) and (ii) can be relaxed to:

(i) for all $M \in \mathrm{Ob}(\mathscr{C} \cap \mathscr{D})$, there exist $n, m \in \mathbb{Z}$ such that $H^{i}(C \cdot(M))=0$ for $i \notin[n, m]$;

(ii) $\# H^{i}(C \cdot(M))$ is finite for all $M \in \mathrm{Ob}(\mathscr{C} \cap \mathscr{D})$.

We shall be interested mostly in the case where $\mathscr{B}=\mathbb{Z}_{p}[[G]]$ is the profinite group algebra of a profinite group $G$. In this case, $\mathscr{B}$ will have only finitely many simple modules if $G$ contains a pro-p group of finite index.

Proof of Proposition 3.1. Let $n, m$ be as in (i). Then the truncated complex

$$
0 \longrightarrow \operatorname{ker}\left(\delta^{n-1}\right) \longrightarrow C^{n-1}(M) \stackrel{\delta^{n-1}}{\longrightarrow} C^{n}(M) \longrightarrow \cdots
$$

is quasi-isomorphic to $C^{\bullet}(M)$, and still satisfies (iii) since $\operatorname{ker}\left(\delta^{n-1}\right)=\operatorname{coker}\left(\delta^{n-3}\right)$ is both a left and a right exact functor in $M$. So from now on we assume that $C^{\bullet}(M)$ is bounded below.

Let $Q_{i}$ be the (projective) object of $\mathscr{C}$ which represents the functor $C^{i}$ and which exists by Grothendieck's theorem. Using a Yoneda Lemma type argument, we obtain a bounded below (chain) complex $\cdots \longrightarrow Q_{i} \longrightarrow Q_{i-1} \longrightarrow \cdots \longrightarrow 0$ in $\mathscr{C}$, and an isomorphism of complexes

$$
\operatorname{Hom}_{\mathscr{B}}^{c}(Q ., M) \cong C^{\bullet}(M)
$$

for all finite $M$. We shall now successively replace the terms in $Q$. by finitely generated projective $\mathscr{B}$-modules. 
Lemma 3.1. Suppose that $Q_{0}^{(n)} \stackrel{\sim}{\longrightarrow} Q$. is a quasi-isomorphism, all $Q_{i}^{(n)}$ are projective, and $Q_{i}^{(n)}$ is finitely generated for $i<n$. Then there is a quasi-isomorphism $Q_{.}^{(n+1)} \stackrel{\sim}{\longrightarrow} Q_{\bullet}^{(n)}$ with $Q_{i}^{(n+1)}=Q_{i}^{(n)}$ for $i<n, Q_{i}^{(n+1)}$ finitely generated for $i<n+1$, and all $Q_{i}^{(n+1)}$ projective.

Proof. We first remark that since any quasi-isomorphism between bounded below complexes of projective objects is a homotopy equivalence, all complexes $\operatorname{Hom}_{\mathscr{B}}^{c}\left(Q_{\bullet}^{(k)}, M\right)$ are quasi-isomorphic to $C^{\bullet}(M)$. Denoting differentials in $Q_{\bullet}^{(n)}$ by $d$, we find an isomorphism

$$
\operatorname{ker}\left(\operatorname{Hom}_{\mathscr{B}}^{c}\left(Q_{n}^{(n)}, M\right) \longrightarrow \operatorname{Hom}_{\mathscr{B}}^{c}\left(Q_{n+1}^{(n)}, M\right)\right) \cong \operatorname{Hom}_{\mathscr{B}}^{c}\left(Q_{n}^{(n)} / \operatorname{im}\left(d_{n+1}\right), M\right)
$$

and an exact sequence

$$
\operatorname{Hom}_{\mathscr{B}}^{c}\left(Q_{n-1}^{(n)}, M\right) \stackrel{\kappa}{\longrightarrow} \operatorname{Hom}_{\mathscr{B}}^{c}\left(Q_{n}^{(n)} / \operatorname{im}\left(d_{n+1}\right), M\right) \longrightarrow H^{n}\left(\operatorname{Hom}_{\mathscr{B}}^{c}\left(Q_{\bullet}^{(n)}, M\right)\right) \longrightarrow 0 .
$$

Since $Q_{n-1}^{(n)}$ is finitely generated, Lemma 3.3 below implies that there is a constant $\beta$ such that \# $\operatorname{Hom}_{\mathscr{B}}^{c}\left(Q_{n-1}^{(n)}, M\right) \leqslant(\# M)^{\beta}$, and by assumption (ii) in Proposition 3.1, we have

$$
\# H^{n}\left(\operatorname{Hom}_{\mathscr{B}}^{c}\left(Q_{\bullet}^{(n)}, M\right)\right)=\# H^{n}\left(C^{\bullet}(M)\right) \leqslant(\# M)^{a_{n}} .
$$

From (3) we deduce \# $\operatorname{Hom}_{\mathscr{B}}^{c}\left(Q_{n}^{(n)} / \operatorname{im}\left(d_{n+1}\right), M\right) \leqslant(\# M)^{\beta+a_{n}}$, hence by Lemma 3.3, $Q_{n}^{(n)} / \operatorname{im}\left(d_{n+1}\right)$ is finitely generated over $\mathscr{B}$. We pick a surjection $Q_{n}^{(n+1)} \rightarrow Q_{n}^{(n)} / \operatorname{im}\left(d_{n+1}\right)$ where $Q_{n}^{(n+1)}$ is finitely generated and projective. This surjection can be lifted to a map $Q_{n}^{(n+1)} \rightarrow Q_{n}^{(n)}$, and we arrive at the following commutative diagram.

$$
\begin{aligned}
\cdots \longrightarrow Q_{n+1}^{(n)} \longrightarrow Q_{n}^{(n)} & \longrightarrow Q_{n-1}^{(n)} \longrightarrow Q_{n-2}^{(n)} \longrightarrow \cdots \\
\uparrow &
\end{aligned}
$$

Here (I) $Q_{i}^{(n+1)}$ is projective for $i \leqslant n$, (II) the vertical map induces an isomorphism in homology for $i<n$, and (III) the vertical map induces a surjection $\operatorname{ker}\left(d_{n}^{\prime}\right) \rightarrow$ $H_{n}\left(Q_{\bullet}^{(n)}\right)$. The conditions (I)-(III) are the inductive assumptions in the proof of [8, Proposition 11.9.1], with $K^{\prime \prime}$ (respectively $K^{\prime}$ ) the class of projective (respectively all) objects in $\mathscr{C}$. Applying this proof, we can inductively complete (4) to a quasiisomorphism $Q_{\bullet}^{(n+1)} \stackrel{\sim}{\longrightarrow} Q_{\bullet}^{(n)}$ as specified in the lemma (where, however, $Q_{i}^{(n+1)}$ need not be finitely generated for $i>n$ ).

Lemma 3.2. If $H^{n}\left(C^{\bullet}(M)\right)=0$ for all finite $\mathscr{B}$-modules $M$, then $H_{n}(Q)=$.0 .

Proof. The exact sequence

$$
0 \longrightarrow \frac{\operatorname{ker} d_{n}}{\operatorname{im} d_{n+1}} \longrightarrow \frac{Q_{n}}{\operatorname{im} d_{n+1}} \longrightarrow \frac{Q_{n}}{\operatorname{ker} d_{n}} \longrightarrow 0
$$

induces an exact sequence

$$
0 \longrightarrow \operatorname{Hom}_{\mathscr{B}}^{c}\left(\frac{Q_{n}}{\operatorname{ker} d_{n}}, M\right) \stackrel{l}{\longrightarrow} \operatorname{Hom}_{\mathscr{B}}^{c}\left(\frac{Q_{n}}{\operatorname{im} d_{n+1}}, M\right) \longrightarrow \operatorname{Hom}_{\mathscr{B}}^{c}\left(\frac{\operatorname{ker} d_{n}}{\operatorname{im} d_{n+1}}, M\right),
$$

and the map $\kappa$ in (3) factors through 1 . So if $H^{n}(C \cdot(M))=H^{n}\left(\operatorname{Hom}_{\mathscr{B}}^{c}\left(Q_{\bullet}^{(n)}, M\right)\right)=0$, 
then we must have $\operatorname{im}(\kappa)=\operatorname{im}(l)=\operatorname{Hom}_{\mathscr{B}}^{c}\left(Q_{n} /\left(\operatorname{im} d_{n+1}\right), M\right)$ or, in other words, that any $\phi \in \operatorname{Hom}_{\mathscr{B}}^{c}\left(Q_{n} /\left(\operatorname{im} d_{n+1}\right), M\right)$ restricts to zero in $\operatorname{Hom}_{\mathscr{B}}^{c}\left(\left(\operatorname{ker} d_{n}\right) /\left(\operatorname{im} d_{n+1}\right), M\right)$. Taking $\phi$ to be the natural projection onto

$$
M:=\left(Q_{n} /\left(\operatorname{im} d_{n+1}\right)\right) / U
$$

where $U$ runs through a fundamental system of neighbourhoods of 0 in $Q_{n} /\left(\operatorname{im} d_{n+1}\right)$, we deduce

$$
H_{n}(Q .)=\frac{\operatorname{ker} d_{n}}{\operatorname{im} d_{n+1}} \subseteq \bigcap U=0 .
$$

Remark 3.2. Unless $\mathscr{B}$ is Noetherian, $H_{n}(Q$.$) need not be finitely generated$ over $\mathscr{B}$.

Proof of Proposition 3.1, continued. Since $Q$. is bounded below, we may put $Q_{.}^{(n)}:=Q$. for some $n \ll 0$, and apply Lemma 3.1 inductively to arrive at a complex $Q_{.}^{(\infty)} \stackrel{\sim}{\longrightarrow} Q$. consisting of finitely generated projective $\mathscr{B}$-modules and which is quasiisomorphic to $Q$. In fact, it suffices to stop at $Q^{(m+2)}$, where $m$ is as in assumption (i) of Proposition 3.1. By Lemma 3.2, the complex $Q$. (and hence the complex $Q_{\text {. }}^{(k)}$ for any $k$ ) is then acyclic in degrees greater than $m$. Define $P$. to be the truncated complex

$$
0 \longrightarrow \operatorname{im}\left(d_{m+1}\right) \longrightarrow Q_{m}^{(\infty)} \longrightarrow Q_{m-1}^{(\infty)} \longrightarrow \cdots \longrightarrow 0 .
$$

We have a projective resolution

$$
\cdots \longrightarrow Q_{m+2}^{(\infty)} \longrightarrow Q_{m+1}^{(\infty)} \longrightarrow \operatorname{im}\left(d_{m+1}\right) \longrightarrow 0
$$

of $\operatorname{im}\left(d_{m+1}\right)$. Since

$$
\operatorname{Ext}_{\mathscr{C}}^{i}\left(\operatorname{im}\left(d_{m+1}\right), M\right)=H^{i}\left(\operatorname{Hom}_{\mathscr{B}}^{c}\left(Q_{\bullet+m+1}^{(\infty)}, M\right)\right) \cong H^{i+m+1}\left(C^{\bullet}(M)\right)=0
$$

for $i>0$ and all finite $M$, we find that $\operatorname{im}\left(d_{m+1}\right)$ is projective in $\mathscr{C}$, again using [2, Proposition 3.1]. Since $Q_{m+1}^{(\infty)}$ is finitely generated over $\mathscr{B}$, so is $\operatorname{im}\left(d_{m+1}\right)$. The natural quasi-isomorphism $Q_{\bullet}^{(\infty)} \stackrel{\sim}{\longrightarrow} P$. is a homotopy equivalence, so we have natural quasi-isomorphisms

$$
\operatorname{Hom}_{\mathscr{B}}(P, M) \longrightarrow \operatorname{Hom}_{\mathscr{B}}^{c}\left(Q_{\bullet}^{(\infty)}, M\right) \longleftarrow \operatorname{Hom}_{\mathscr{B}}^{c}(Q ., M) \longrightarrow C^{\bullet}(M) .
$$

The complex $P$. therefore satisfies all requirements. The converse statement in Proposition 3.1 follows easily from the fact that $P$. is bounded, together with Lemma 3.3 below.

In the remainder of this section, we briefly discuss the extent to which the representability result of Proposition 3.1 is valid on larger categories.

Proposition 3.2. Suppose that $C \cdot$ in Proposition 3.1 is the restriction of a functor on $\mathscr{C}$ (respectively $\mathscr{D}$ ) with values in the category of complexes of $\mathbb{Z}_{p}$-modules. Assume also that $C^{\bullet}$ commutes with filtered inverse (respectively direct) limits. Then there is an isomorphism of functors from $\mathscr{C}$ (respectively $\mathscr{D}$ ) into the derived category of $\mathbb{Z}_{p^{-}}$ modules

$$
\operatorname{Hom}_{\mathscr{B}}\left(P_{\bullet},-\right) \stackrel{\sim}{\longrightarrow} C \cdot(-),
$$

where $P_{.}$is as in Proposition 3.1. 
Proof. The first two maps in (6) are quasi-isomorphisms for any object $M$ of either $\mathscr{C}$ or $\mathscr{D}$, because they are induced by homotopy equivalences of complexes. To show that the third map is a quasi-isomorphism, represent $M$ as a filtered inverse (respectively direct) limit

$$
M=\lim _{\longleftarrow} M_{l} \quad\left(\text { respectively } M=\underset{\lim }{\longrightarrow} M_{\imath}\right)
$$

of objects $M_{l}$ of $\mathscr{C} \cap \mathscr{D}$, and use the fact that both $\operatorname{Hom}_{\mathscr{B}}^{c}\left(Q_{i},-\right)$ and $C^{i}(-)$ commute with these limits. In the case of $\operatorname{Hom}_{\mathscr{B}}^{c}\left(Q_{i},-\right)$, this is by definition of an inverse limit (respectively by [2, Lemma A.3] for the direct limit).

In the situation of Proposition 3.2, the functor $\operatorname{Hom}_{\mathscr{B}}\left(P_{.},-\right)$on $\mathscr{C}$ (respectively $\mathscr{D}$ ) actually takes values in the bounded derived category of the abelian category $\mathscr{C}\left(\mathbb{Z}_{p}\right)$ (respectively $\mathscr{D}\left(\mathbb{Z}_{p}\right)$ ), as is easily seen by representing $M$ as in (7). Let $D$ be the derived category of $\mathscr{C}$ (respectively $\mathscr{D}$ ), and let $D\left(\mathbb{Z}_{p}\right)$ be the derived category of $\mathscr{C}\left(\mathbb{Z}_{p}\right)$ (respectively $\mathscr{D}\left(\mathbb{Z}_{p}\right)$ ). A functor $C^{\bullet}$ as in Proposition 3.2 naturally extends to a functor $F: D \longrightarrow D\left(\mathbb{Z}_{p}\right)$ by sending $M^{\bullet}$ to the total complex of $C^{\bullet}\left(M^{\bullet}\right)$ or, equivalently, of $\operatorname{Hom}_{\mathscr{B}}^{c}\left(P_{\bullet}, M^{\bullet}\right)$. $F$ will satisfy properties (i) and (ii) of Proposition 3.1 when considered on $\mathscr{C} \cap \mathscr{D}$, via the natural full embedding $\mathscr{C} \cap \mathscr{D} \longrightarrow D$. $F$ will also be exact in the sense of being a triangulated functor.

Question. Conversely, is every triangulated functor $F: D \longrightarrow D\left(\mathbb{Z}_{p}\right)$ which satisfies (i) and (ii) of Proposition 3.1 of the form $M^{\bullet} \longmapsto \operatorname{Hom}^{\bullet}\left(P_{\bullet}, M^{\bullet}\right)$ for some bounded complex $P$. of finitely generated projective $\mathscr{B}$-modules? A positive answer to this question would be important in situations where one does not have 'standard resolutions'.

Lemma 3.3. The following are equivalent for an object $M$ of $\mathscr{C}$.

(a) $\quad M$ is finitely generated over $\mathscr{B}$.

(b) There is a constant $a \geqslant 0$ such that $\# \operatorname{Hom}_{\mathscr{B}}^{c}(M, X) \leqslant(\# X)^{a}$ for all finite $\mathscr{B}$-modules $X$.

(c) There is $a \geqslant 0$ such that $\operatorname{dim}_{D} \operatorname{Hom}_{\mathscr{B}}^{c}(M, X) \leqslant a \cdot \operatorname{dim}_{D} X$ for all simple $\mathscr{B}$-modules $X$. Here $D$ is the finite field $\operatorname{End}_{\mathscr{B}} X$.

Proof. (a) $\Rightarrow$ (b). If $M$ is finitely generated by $m_{1}, \ldots, m_{r}$ as a $\mathscr{B}$-module, then any $\phi \in \operatorname{Hom}_{\mathscr{B}}^{c}(M, X)$ is uniquely determined by $\left(\phi\left(m_{1}\right), \ldots, \phi\left(m_{r}\right)\right) \in X^{r}$. Hence $\# \operatorname{Hom}_{\mathscr{B}}^{c}(M, X) \leqslant \# X^{r}=(\# X)^{r}$.

(b) $\Rightarrow$ (c). Choosing $X$ to be simple, and taking logarithms to the base $\# D$ on both sides of (b), gives $\operatorname{dim}_{D} \operatorname{Hom}_{\mathscr{B}}^{c}(M, X) \leqslant a \cdot \operatorname{dim}_{D} X$.

(c) $\Rightarrow$ (a). First we make use of the fact that any object $M$ of $\mathscr{C}$ has a projective hull, that is, a homomorphism $\phi: P \longrightarrow M$, with $P$ projective, $\phi(P)=M$, and $\phi(N) \neq M$ for all proper closed submodules $N$ of $P$. For Artinian $\mathscr{B}$, this is proved in [16, Proposition 41], and the general case follows by noting that for any surjection $B \rightarrow B^{\prime}$ of Artinian rings and projective hull $P \longrightarrow M, P \otimes_{B} B^{\prime} \longrightarrow M \otimes_{B} B^{\prime}$ is a projective hull. Using then the fact that projective hulls are unique up to isomorphism we obtain, for general profinite $\mathscr{B}$, an inverse system of projective hulls over the finite quotients of $\mathscr{B}$ whose inverse limit is projective in $\mathscr{C}$ [2, Corollary 3.3]. If $X$ is simple, then it follows easily from the definition that a projective hull $\phi$ induces a bijection

$$
\operatorname{Hom}_{\mathscr{B}}^{c}(M, X) \stackrel{\sim}{\longrightarrow} \operatorname{Hom}_{\mathscr{B}}^{c}(P, X) .
$$


Now we make use of the structure theorem for projective objects in $\mathscr{C}$ as given in [7, Chapter V, Section 2, Theorem 4.5 and Example 4.6b)], which also follows from the corresponding theorem over Artinian rings [16, Corollary to Proposition 41]. Let $\Sigma$ be a set of representatives for the isomorphism classes of simple $\mathscr{B}$-modules, and for each $S \in \Sigma$ choose a projective hull $P_{S} \longrightarrow S$. For any projective object $P$ of $\mathscr{C}$, there exist index sets $I_{S}(P)$, of cardinality uniquely determined by $P$, and an isomorphism of objects in $\mathscr{C}$

$$
P \cong \prod_{S \in \Sigma} \prod_{I_{S}(P)} P_{S}
$$

where the right-hand side carries the product topology. In particular, if $X \in \Sigma$, $D:=\operatorname{End}_{\mathscr{B}} X$, then we find, using (8) with $M=S \in \Sigma$,

$$
\operatorname{Hom}_{\mathscr{B}}^{c}(P, X)=\bigoplus_{S \in \Sigma} \bigoplus_{I_{S}(P)} \operatorname{Hom}_{\mathscr{B}}^{c}\left(P_{S}, X\right)=\bigoplus_{S \in \Sigma} \bigoplus_{I_{S}(P)} \operatorname{Hom}_{\mathscr{B}}^{c}(S, X)=\bigoplus_{I_{X}(P)} D .
$$

This formula applied to $P=\mathscr{B}$ gives

$$
X \cong \operatorname{Hom}_{\mathscr{B}}^{c}(\mathscr{B}, X)=\bigoplus_{I_{X}(\mathscr{B})} D,
$$

and we find that the set $I_{X}(\mathscr{B})$ is finite (for any $X \in \Sigma$ ) because $X$ is finite. Using (8), (9), (10) and the assumption in (c), we obtain

$$
\# I_{X}(P)=\operatorname{dim}_{D} \operatorname{Hom}_{\mathscr{B}}^{c}(P, X)=\operatorname{dim}_{D} \operatorname{Hom}_{\mathscr{B}}^{c}(M, X) \leqslant a \cdot \operatorname{dim}_{D} X=a \cdot \# I_{X}(\mathscr{B}) .
$$

Hence we can choose an injection of sets $I_{X}(P) \hookrightarrow \coprod_{a \text { copies }} I_{X}(\mathscr{B})$, and obtain a surjection in $\mathscr{C}$

$$
\left(\prod_{I_{X}(\mathscr{B})} P_{X}\right)^{a} \longrightarrow \prod_{I_{X}(P)} P_{X}
$$

After taking the product over all $X \in \Sigma$, we find a surjection

$$
\mathscr{B}^{a}=\left(\prod_{S \in \Sigma} \prod_{I_{S}(\mathscr{B})} P_{S}\right)^{a} \longrightarrow \prod_{S \in \Sigma} \prod_{I_{S}(P)} P_{S}=P \stackrel{\phi}{\longrightarrow} M,
$$

which shows that $M$ is finitely generated.

REMARK 3.3. As in Remark 3.1 above, if $\Sigma$ is finite, then conditions (b) and (c) in Lemma 3.3 can be relaxed to the following.

$\left(\mathrm{b}^{\prime}\right) \operatorname{Hom}_{\mathscr{B}}^{c}(M, X)$ is finite for all finite $\mathscr{B}$-modules $X$.

(c') $\operatorname{dim}_{D} \operatorname{Hom}_{\mathscr{B}}^{c}(M, X)$ is finite for all simple $\mathscr{B}$-modules $X$, where $D=\operatorname{End}_{\mathscr{B}} X$.

See also [13] for a proof of Lemma 3.3 in this case.

\section{Computing the equivariant Euler characteristic}

Let $\mathscr{A}$ be as in Section 2 , and let $\mathscr{B}$ be as in Section 3 . In this section we shall consider objects $M$ of $\mathscr{C}$ which also carry an $\mathscr{A}$-action commuting with the $\mathscr{B}$-action, in other words, $\mathscr{A} \otimes_{\mathbb{Z}_{p}} \mathscr{B}$-modules. We shall assume throughout that $M$ is finitely generated over $\mathscr{A}$ (and hence over $\mathbb{Z}_{p}$ ). 
Proposition 4.1. Assume that $M \longmapsto C^{\bullet}(M)$ is a functor as in Proposition 3.1, induced from a functor on $\mathscr{C}$ as in Proposition 3.2.

(a) $C \cdot$ maps $\mathscr{A} \otimes_{\mathbb{Z}_{p}} \mathscr{B}$-modules of finite projective $\mathscr{A}$-dimension to perfect complexes of $\mathscr{A}$-modules, that is, objects of $D^{\text {perf }}(\mathscr{A})$.

(b) $C \cdot$ maps finite $\mathscr{A} \otimes_{\mathbb{Z}_{p}} \mathscr{B}$-modules of finite projective $\mathscr{A}$-dimension to objects of $D^{f \operatorname{perf}}(\mathscr{A})$.

(c) Suppose, in addition, that $[C \cdot(M)]=d[M]$ in $K_{0}\left(\mathbb{Z}_{p}, \mathbb{Q}_{p}\right)$ for some integer $d$ and all finite $M$. Then $\left[C^{\bullet}(M)\right]_{\mathscr{A}}=d[M]_{\mathscr{A}}$ in $K_{0}\left(\mathscr{A}, \mathbb{Q}_{p}\right)$ for all finite $\mathscr{A} \otimes_{\mathbb{Z}_{p}} \mathscr{B}$-modules $M$ of finite projective $\mathscr{A}$-dimension.

Proof. We use the notation of the proof of Lemma 3.3 and the structure theorem for projective objects of $\mathscr{C}$ mentioned there. If $M$ is a (left) $\mathscr{A} \otimes_{\mathbb{Z}_{p}} \mathscr{B}$-module and $P$ is an object of $\mathscr{C}$, then $\operatorname{Hom}_{\mathscr{C}}(P, M)=\operatorname{Hom}_{\mathscr{B}}^{c}(P, M)$ retains a (left) $\mathscr{A}$-action. For any simple module $S \in \Sigma$, put $M_{S}=\operatorname{Hom}_{\mathscr{B}}^{c}\left(P_{S}, M\right)$.

Lemma 4.1. We have $M_{S}=0$ for all but finitely many $S \in \Sigma$.

Proof. The action of $\mathscr{B}$ on $M$ factors through the algebra

$$
B^{\prime}=\operatorname{im}\left(B \rightarrow \operatorname{End}_{\mathscr{A}} M\right),
$$

which is finitely generated as a $\mathbb{Z}_{p}$-module and hence has only finitely many simple modules up to isomorphism. If $S \in \Sigma$ is not one of those simple $B^{\prime}$-modules, then we have

$$
M_{S}=\operatorname{Hom}_{\mathscr{B}}^{c}\left(P_{S}, M\right)=\lim _{\longleftarrow} \operatorname{Hom}_{\mathscr{B}}^{c}\left(P_{S}, M \otimes_{B} B^{\prime \prime}\right),
$$

where $B^{\prime \prime}$ runs through the finite quotients of $B^{\prime}$. Choosing a composition series of the finite $B^{\prime}$-module $M \otimes_{B} B^{\prime \prime}$, none of its simple subquotients will be isomorphic to $S$, hence $\operatorname{Hom}_{\mathscr{B}}^{c}\left(P_{S}, M \otimes_{B} B^{\prime \prime}\right)=0$ by an easy inductive argument using (8).

Proof of Proposition 4.1, continued. Similarly to (10), there is a direct sum decomposition of $\mathscr{A}$-modules

$$
M \cong \operatorname{Hom}_{\mathscr{B}}^{c}(\mathscr{B}, M) \cong \bigoplus_{S \in \Sigma} \bigoplus_{I_{S}(\mathscr{B})} M_{S},
$$

where the right-hand direct sum is in fact finite, by Lemma 4.1. If $M$ has finite projective dimension over $\mathscr{A}$, then the direct summand $M_{S}$ of $M$ will also have finite projective dimension. Now consider the complex $P$. of Proposition 3.1. Since

$$
\operatorname{Hom}_{\mathscr{B}}^{c}\left(P_{i}, M\right)=\bigoplus_{S \in \Sigma} \bigoplus_{I_{S}\left(P_{i}\right)} M_{S}
$$

the complex $\operatorname{Hom}_{\mathscr{B}}^{c}\left(P_{\bullet}, M\right) \cong C \cdot(M)$ is a bounded complex of finitely generated $\mathscr{A}$-modules of finite projective $\mathscr{A}$-dimension, hence perfect. This gives (a). If $M$ is finite, then so are the $M_{S}$, which gives (b). To prove (c), note that

$$
\left[C^{\bullet}(M)\right]_{\mathscr{A}}=\sum_{i \in \mathbb{Z}}(-1)^{i} \sum_{S \in \Sigma} \# I_{S}\left(P_{i}\right)\left[M_{S}\right]_{\mathscr{A}}=\sum_{S \in \Sigma}\left(\sum_{i \in \mathbb{Z}}(-1)^{i} \# I_{S}\left(P_{i}\right)\right)\left[M_{S}\right]_{\mathscr{A}}
$$


If $M \in \Sigma$, then we have $M_{S}=0$ for $S \neq M$, by (8). Taking $\mathscr{A}=\mathbb{Z}_{p}$ and $M \in \Sigma$ in (13), the assumption in (c) gives

$$
d[M]=\sum_{i \in \mathbb{Z}}(-1)^{i} \# I_{M}\left(P_{i}\right)\left[M_{M}\right],
$$

and (11) yields $[M]=\left(\# I_{M}(\mathscr{B})\right)\left[M_{M}\right]$ for $M \in \Sigma$. We conclude that

$$
\sum_{i \in \mathbb{Z}}(-1)^{i} \# I_{M}\left(P_{i}\right)=d \cdot \# I_{M}(\mathscr{B})
$$

for all $M \in \Sigma$, since $\left[M_{M}\right] \neq 0$ in $K_{0}\left(\mathbb{Z}_{p}, \mathbb{Q}_{p}\right) \cong \mathbb{Z}$. Together with (11) and (13), this yields (c).

Remark 4.1. It is clear from the proof of Proposition 4.1 that any other 'explicit' formula for the Euler characteristic of $C \cdot(M)$ would determine the integers

$$
\sum_{i \in \mathbb{Z}}(-1)^{i} \# I_{S}\left(P_{i}\right)
$$

and hence the equivariant Euler characteristic $\left[C^{\bullet}(M)\right]_{\mathscr{A}}$.

We conclude this section with another typical application of Proposition 3.1.

Proposition 4.2. Assume that $M \longmapsto C^{\bullet}(M)$ is a functor as in Proposition 3.1, induced from a functor on $\mathscr{C}$ as in Proposition 3.2. Let $\mathscr{A} \longrightarrow \mathscr{A}^{\prime}$ be a homomorphism of algebras satisfying the assumptions of Section 2, and let $M$ be an $\mathscr{A} \otimes_{\mathbb{Z}_{p}} \mathscr{B}$-module, finitely generated and projective over $\mathscr{A}$. Then the natural map

$$
C \cdot(M) \otimes \mathscr{A}^{L} \mathscr{A}^{\prime} \longrightarrow C^{\bullet}\left(M \otimes_{\mathscr{A}} \mathscr{A}^{\prime}\right)
$$

is a quasi-isomorphism.

Proof. By (11) and (12), the complex $\operatorname{Hom}_{\mathscr{B}}\left(P_{\bullet}, M\right)$ is a bounded complex of finitely generated projective $\mathscr{A}$-modules. Hence the quasi-isomorphism

$$
\operatorname{Hom}_{\mathscr{B}}\left(P_{\bullet}, M\right) \cong C^{\bullet}(M)
$$

of Proposition 3.1 is a flat resolution of $C^{\bullet}(M)$, and by definition $C^{\bullet}(M) \otimes_{\mathscr{A}}^{L} \mathscr{A}^{\prime} \cong$ $\operatorname{Hom}_{\mathscr{B}}(P, M) \otimes_{\mathscr{A}} \mathscr{A}^{\prime}$. But the natural map

$$
\operatorname{Hom}_{\mathscr{B}}(P, M) \otimes_{\mathscr{A}} \mathscr{A}^{\prime} \longrightarrow \operatorname{Hom}_{\mathscr{B}}\left(P ., M \otimes_{\mathscr{A}} \mathscr{A}^{\prime}\right) \cong C^{\bullet}\left(M \otimes_{\mathscr{A}} \mathscr{A}^{\prime}\right)
$$

is clearly a quasi-isomorphism: one reduces to $P$ a free $\mathscr{B}$-module and then to $P=\mathscr{B}$, in which case the map $\operatorname{Hom}_{\mathscr{B}}(P, M) \otimes_{\mathscr{A}} \mathscr{A}^{\prime} \longrightarrow \operatorname{Hom}_{\mathscr{B}}\left(P, M \otimes_{\mathscr{A}} \mathscr{A}^{\prime}\right)$ is simply the identity of $M \otimes_{\mathscr{A}} \mathscr{A}^{\prime}$.

\section{Examples}

(1) Profinite groups. Suppose that $\Gamma$ is a profinite group such that

(a) $\operatorname{cd}_{p}(\Gamma)<\infty$,

(b) $H^{i}(\Gamma, M)$ is finite for all finite $\mathbb{Z}_{p}$-modules $M$ with continuous $\Gamma$-action.

Assumption (b) is too weak to apply Proposition 3.1 directly to the standard continuous cochain complex $C^{\bullet}(\Gamma, M)$ with $\mathscr{B}=\mathbb{Z}_{p}[[\Gamma]]$ the profinite group algebra of $\Gamma$. However, we have the following. 
Proposition 5.1 (compare [15, Remark after Proposition 3.5.2]). Assume that $\Gamma$ satisfies (a) and (b) above, and that $G$ is a quotient of $\Gamma$ which contains a pro-p subgroup of finite index. Then there exists a bounded complex $P$. of finitely generated projective $\mathbb{Z}_{p}[[G]]$-modules such that $R \Gamma(\Gamma, M) \cong \operatorname{Hom}_{\left.\mathbb{Z}_{p}[G]\right]}(P ., M)$ for all continuous, profinite or discrete, $\mathbb{Z}_{p}[[G]]$-modules $M$.

Proof. For finite $M$, this follows from Proposition 3.1 and Remark 3.1 applied to $\mathscr{B}=\mathbb{Z}_{p}[[G]]$. If one defines the group cohomology $R \Gamma(\Gamma, M)$ for all profinite or discrete $\mathscr{B}$-modules via the standard continuous cochain complex $C \bullet(\Gamma, M)$, then the assumptions of Proposition 3.2 are satisfied, which completes the proof.

REMARK 5.1. If $\mathscr{C}$ denotes the abelian category of profinite $\mathscr{B}=\mathbb{Z}_{p}[[\Gamma]]$-modules considered in Section 3, then $C \cdot(\Gamma, M)$ is quasi-isomorphic to $R \operatorname{Hom}_{\mathscr{C}}\left(\mathbb{Z}_{p}, M\right)$ for any object $M$ of $\mathscr{C}$. Indeed, giving a continuous $\Gamma$-equivariant map $\Gamma \times \cdots \times \Gamma \longrightarrow M$ is equivalent to giving a continuous $\mathbb{Z}_{p}[[\Gamma]]$-homomorphism $F_{n} \longrightarrow M$, where $F_{n}:=\lim _{U} \mathbb{Z}_{p}[\Gamma / U \times \cdots \times \Gamma / U]$ (the limit being taken over all open subgroups $U$ of $\Gamma$ ). By [2, Corollary 3.3], $F_{n}$ is projective in $\mathscr{C}$ because $\mathbb{Z}_{p}[\Gamma / U \times \cdots \times \Gamma / U]$ is a projective (indeed, free) $\mathbb{Z}_{p}[\Gamma / U]$-module. Moreover, the standard boundary maps give a projective resolution $F_{\bullet} \rightarrow \mathbb{Z}_{p}$ in $\mathscr{C}$, so $\operatorname{Hom}_{\mathscr{C}}\left(F_{\bullet}, M\right)=C^{\bullet}(\Gamma, M)$.

Proposition 5.2. Assume that $\Gamma$ satisfies (a) and (b), and that $M$ is a continuous $\mathscr{A}[[\Gamma]]-m o d u l e$, finitely generated over $\mathscr{A}$. If $M$ has finite projective dimension over $\mathscr{A}$ (and is finite), then $R \Gamma(\Gamma, M)$ is an object of $D^{\text {perf }}(\mathscr{A})$ (respectively $D^{f \text { perf }}(\mathscr{A})$ ). If $[R \Gamma(\Gamma, M)]=d[M]$ for all finite $M$, then $[R \Gamma(\Gamma, M)]_{\mathscr{A}}=d[M]_{\mathscr{A}}$ for all finite $M$ of finite projective $\mathscr{A}$-dimension.

Proof. This follows by applying Proposition 4.1 to $\mathscr{B}:=\mathbb{Z}_{p}[[G]]$ and $C^{\bullet}(-):=$ $C \cdot(\Gamma,-)$, where $G=\operatorname{im}\left(\Gamma \rightarrow \operatorname{Aut}_{\mathscr{A}}(M)\right)$. Since $M$ is finitely generated over $\mathbb{Z}_{p}$, $G$ contains a pro-p subgroup of finite index, and Remark 3.1 applies.

(2) Local fields. Let $\Gamma$ be the absolute Galois group of a finite extension $K$ of $\mathbb{Q}_{l}$. Then (a) and (b) of Example (1) hold for $\Gamma$, and we also know that

$$
[R \Gamma(\Gamma, M)]=-\left[K: \mathbb{Q}_{l}\right] \delta_{l, p}[M]
$$

for finite continuous $\mathbb{Z}_{p}[[\Gamma]]$-modules $M$, where $\delta_{l, p}$ is the Kronecker delta [14, Theorem II.5]. Hence Propositions 5.1 and 5.2 apply. In fact, one can show slightly more.

Proposition 5.3. Let $\Gamma$ be the absolute Galois group of a finite extension $K$ of $\mathbb{Q}_{l}$. Then there exists a bounded complex $P$. of finitely generated, projective $\mathscr{B}:=\mathbb{Z}_{p}[[\Gamma]]-$ modules such that $R \Gamma(\Gamma, M)=\operatorname{Hom}_{\mathscr{B}}^{c}\left(P_{\bullet}, M\right)$ for all continuous, profinite or discrete, $\mathscr{B}$-modules $M$.

Proof. One has the estimates

$$
\# H^{0}(\Gamma, M) \leqslant \# M, \quad \# H^{2}(\Gamma, M)=\# H^{0}\left(\Gamma, M^{*}(1)\right) \leqslant \# M^{*}(1)=\# M
$$

by local duality [14, Theorem II.2], and

$$
\# H^{1}(\Gamma, M)=\# H^{0}(\Gamma, M) \# H^{2}(\Gamma, M) \# M^{\left[K: \mathbb{Q}_{]}\right] \delta_{l, p}} \leqslant \# M^{\left[K: \mathbb{Q}_{]}\right] \delta_{l, p}+2}
$$


by (16). Hence Proposition 3.1 applies directly to $\mathscr{B}=\mathbb{Z}_{p}[[\Gamma]]$ and $C^{\bullet}(M)=$ $C \cdot(\Gamma, M)$.

For more examples, see $[\mathbf{1 4}, \mathbf{1 5}]$.

(3) Étale cohomology. Let $X$ be a scheme such that

(a) $\operatorname{cd}_{p}\left(X_{\text {et }}\right)<\infty$,

(b) $H^{i}\left(X_{\text {et }}, \mathscr{F}\right)$ is finite for all constructible sheaves of $\mathbb{Z}_{p}$-modules $\mathscr{F}$ on $X$.

If $R$ is a separably closed or local field of characteristic different from $p$, or $R=\mathbb{Z}\left[p^{-1}\right]$ and $p \neq 2$, then any scheme $X \stackrel{\pi}{\longrightarrow} \operatorname{Spec}(R)$ of finite type over $\operatorname{Spec}(R)$ satisfies (a) and (b). This is an immediate consequence of the Leray spectral sequence for $\pi$, together with the following facts.

- A constructible sheaf of $\mathbb{Z}_{p}$-modules $\mathscr{F}$ on $\operatorname{Spec}(R)$ has finite cohomology and $\operatorname{cd}_{p}(\operatorname{Spec}(R))<\infty[12$, II, Theorem 3.1].

- If $\mathscr{F}$ is constructible on $X$, then the sheaves $R^{i} \pi_{*}(\mathscr{F})$ are constructible on $\operatorname{Spec}(R)$ [6, Finitude, Theorem 1.1].

- $R^{i} \pi_{*}(\mathscr{F})=0$ for constructible $\mathscr{F}$ and $i>N$ (where $i$ is an integer depending on $X$ but not on $\mathscr{F}$ ) [6, Finitude, Remark before 1.4].

Let $Y \longrightarrow X$ be a (profinite) Galois cover of schemes with group $G$, and put $\mathscr{B}=\mathbb{Z}_{p}[[G]]$. Any profinite continuous $\mathscr{B}$-module $M$, finitely generated over $\mathbb{Z}_{p}$, gives rise to a locally constant $\mathbb{Z}_{p}$-adic sheaf on $X$ which we denote by the same letter $M$. (This functor from modules to sheaves is exact.)

Proposition 5.4. Suppose that $X$ is a scheme satisfying (a) and (b), and that $Y \longrightarrow X$ is a Galois cover with group $G$ such that $G$ contains a pro-p subgroup of finite index. Then there exists a bounded complex of finitely generated $\mathscr{B}=\mathbb{Z}_{p}[[G]]-$ modules $P_{\text {., }}$ and a natural quasi-isomorphism

$$
R \Gamma\left(X_{\mathrm{et}}, M\right) \stackrel{\sim}{\longrightarrow} \operatorname{Hom}_{\mathscr{B}}(P ., M)
$$

for all continuous $\mathscr{B}$-modules $M$, finitely generated over $\mathbb{Z}_{p}$.

Proof. Let $M \longrightarrow \mathscr{G} \cdot(M)$ be the Godement resolution of $M$ [11, Remark III.1.20(c)]. Then the complex $C^{\bullet}(M)=H^{0}(X, \mathscr{G} \bullet(M))$ is functorial and exact in $M$, and computes $R \Gamma\left(X_{\mathrm{et}}, M\right)$. This proposition then follows from Proposition 3.1 and Remark 3.1 applied to $\mathscr{B}$ and $C^{\bullet}(M)$.

Proposition 5.5. Assume that $X$ satisfies (a) and (b), and that $M$ is a locally constant sheaf of $\mathscr{A}$-modules on $X$, finitely generated over $\mathscr{A}$. If $M$ has finite projective dimension over $\mathscr{A}$ (and is finite), then $R \Gamma\left(X_{\mathrm{et}}, M\right)$ is an object of $D^{\text {perf }}(\mathscr{A})$ (respectively $\left.D^{f \operatorname{perf}}(\mathscr{A})\right)$. If $\left[R \Gamma\left(X_{\mathrm{et}}, M\right)\right]=d[M]$ for all finite $M$, then $\left[R \Gamma\left(X_{\mathrm{et}}, M\right)\right]_{\mathscr{A}}=d[M]_{\mathscr{A}}$ for all finite $M$ of finite projective $\mathscr{A}$-dimension.

Proof. Results of this type are more or less well known, at least when $\mathscr{A}$ is Artinian [6, Finitude, Remark 1.7, and Rapport, Lemme 4.5.1]. The proof of this proposition is the same as that of Proposition 5.2, using the Godement resolution as in Proposition 5.4.

There are variants of these statements for the cohomology with compact support $R \Gamma_{c}\left(X_{\mathrm{et}}, \mathscr{F}\right)$. 
(4) Global fields. This is the case which gave rise to this note. Suppose that $K$ is a global field of characteristic different from $p$, and that $S$ is a finite set of places of $K$ including the archimedean ones and those dividing $p$. Denote by $G_{S}$ the Galois group of the maximal algebraic extension of $K$ unramified at places not in $S$, and by $G_{v}$ the absolute Galois group of the complete local field $K_{v}$ for $v \in S$. If $M$ is a continuous (profinite or discrete) $G_{S}$-module, put

$$
C \cdot(M)=\text { Cone }\left(C^{\bullet}\left(G_{S}, M\right) \longrightarrow \prod_{v \in S} C^{\bullet}\left(G_{v}, M\right)\right)[-1],
$$

where $C^{\bullet}(-,-)$ is the standard continuous cochain complex as in Example (1) of this section. Then $C^{\bullet}(M)$ is, in fact, quasi-isomorphic to the étale cohomology with compact support $R \Gamma_{c}(U, M)$ considered in $[4,1.9]$, where $U$ is the spectrum of the ring of $S$-integers in $K$.

Theorem 5.1. Suppose that $\mathscr{A}$ is as in Section 2, that $U$ is as above, and that $M$ is a continuous $\mathscr{A}\left[\left[G_{S}\right]\right]-$ module, finitely generated over $\mathscr{A}$ (respectively finite) and of finite projective dimension over $\mathscr{A}$. Then $R \Gamma_{c}(U, M)$ is a perfect complex of $\mathscr{A}$-modules (respectively an object of $D^{f \operatorname{perf}}(\mathscr{A})$, and

$$
\left[R \Gamma_{c}(U, M)\right]_{\mathscr{A}}=0
$$

in $\left.K_{0}\left(\mathscr{A}, \mathbb{Q}_{p}\right)\right)$.

Proof. If we put $\mathscr{B}=\mathbb{Z}_{p}[[G]]$ where $G=\operatorname{im}\left(G_{S} \rightarrow \operatorname{Aut}_{\mathscr{A}}(M)\right)$, then the complex $C^{\bullet}(-)$ defined in (17) satisfies all assumptions of Proposition 4.1. One also knows that $[C \cdot(M)]=0$ in $K_{0}\left(\mathbb{Z}_{p}, \mathbb{Q}_{p}\right)$. (This is an immediate reformulation of Tate's formula for the global Euler characteristic [12, I, Theorem 5.1].)

Remark 5.2. If $\mathscr{A}$ is commutative, then by using Remark 2.1 and the determinant functor as in [5], one can show that $K_{0}\left(\mathscr{A}, \mathbb{Q}_{p}\right)$ is generated by all triples $(X, g, \mathscr{A})$, where $X$ is an invertible $\mathscr{A}$-module and $g: X \otimes_{\mathbb{Z}_{p}} \mathbb{Q}_{p} \cong \mathscr{A}_{\mathbb{Q}}$ is an isomorphism. That is, $K_{0}\left(\mathscr{A}, \mathbb{Q}_{p}\right)$ appears as the group of line bundles on $\operatorname{Spec}(\mathscr{A})$ together with a trivialization on $\operatorname{Spec}\left(\mathscr{A}_{\mathbb{Q}}\right)$. From this point of view, the statement of $[4$, Proposition $1.20(\mathrm{~b})]$ amounts to an identity in $K_{0}\left(\mathbb{Z}_{p}[G], \mathbb{Q}_{p}\right)$, and Theorem 5.1 is a direct generalization of [4, Proposition 1.20(b)] to any algebra $\mathscr{A}$ as in Section 2.

If $\mathscr{A}$ is Artinian and the Cartan map

$$
K_{0}(\mathscr{A}[G]) \longrightarrow G_{0}(\mathscr{A}[G])
$$

is injective for finite groups $G$, then Theorem 5.1 can be proved along the lines of the proof of [4, Proposition 1.20]. However, in [3] one needs the case where $\mathscr{A}$ is an order in a finite dimensional semisimple $\mathbb{Q}_{p}$-algebra. There does not seem to be a straightforward way to deduce the general case from the Artinian case. Also, there are Artinian rings for which the Cartan map is not injective.

Question. Do the hypotheses of Proposition 3.1 hold for $\mathscr{B}=\mathbb{Z}_{p}\left[\left[G_{S}\right]\right]$ and $C^{\bullet}(M)$ as in (17) (or $C^{\bullet}(M)=C^{\bullet}\left(G_{S}, M\right.$ ) if $p \neq 2$ )? This question was raised by J-P. Serre in discussions with the author. He also expressed his belief that the answer to the question is, in fact, negative. 


\section{References}

1. H. Bass, Algebraic K-theory (Benjamin, New York, 1968).

2. A. Brumer, 'Pseudocompact algebras, profinite groups and class formations', J. Algebra 4 (1966) $442-470$.

3. D. BuRns, 'Iwasawa theory and $p$-adic Hodge theory over non-commutative algebras I', Preprint, 1997.

4. D. Burns and M. FlaCh, 'Motivic L-functions and Galois module structures', Math. Ann. 305 (1996) $65-102$.

5. D. Burns and M. FlaCh, 'Equivariant Tamagawa numbers of motives', Preprint, 1998.

6. P. Deligne, Cohomologie étale, Lecture Notes in Math. 569 (Springer, New York, 1977).

7. M. Demazure and P. Gabriel, Groupes algébriques (Masson, Paris, 1970).

8. A. GrothendiecK, 'Eléments de géometrie algébrique', Publ. Math. IHES 11 (1961).

9. A. GrothendiecK, 'Technique de descent et théorèmes d'existence en géometrie algébrique II', Sém. Bourbaki 195 (1959/60).

10. A. Grothendieck, Cohomologie l-adique et fonctions L, SGA 5, Lecture Notes in Math. 589 (Springer, New York, 1977).

11. J. S. MiLne, Étale cohomology (Princeton University Press, 1980)

12. J. S. Milne, Arithmetic duality theorems, Perspect. Math. 1 (Academic Press, Boston, MA, 1986).

13. A. Pletch, 'Profinite duality groups I', J. Pure Appl. Algebra 16 (1980) 55-74.

14. J-P. Serre, Cohomologie Galoisienne, Lecture Notes in Math. 5 (Springer, New York, 1964).

15. J-P. SERRE, 'La distribution d'Euler-Poincaré d'un groupe profini', Galois representations in arithmetic algebraic geometry, London Math. Soc. Lecture Note Ser. 254 (ed. A. J. Scholl and R. L. Taylor, Cambridge University Press, 1998).

16. J-P. SerRe, Linear representations of finite groups, Grad. Texts in Math. 42 (Springer, New York, 1977).

17. J. TATE, 'Duality theorems in Galois cohomology over number fields', Proc. Internat. Congr. Math., Stockholm 1962 (Institut Mittag-Leffler, Djursholm, Sweden, 1963) 288-295.

18. J. TATE, On the conjectures of Birch and Swinnerton-Dyer and a geometric analog, Sém. Bourbaki 306 (Benjamin, New York, 1966).

19. R. W. ThOmASON and T. TrobaUGH, 'Higher algebraic K-theory of schemes and of derived categories', The Grothendieck Festschrift, Vol. III (ed. P. Cartier et al., Birkhäuser, Boston, 1990).

\section{Department of Mathematics}

Caltech

Pasadena, CA 91125

USA 